\title{
RESTRICCIÓN DEL CRECIMIENTO SELECTIVO INTRA UTERINO Y RIESGO DE MORTALIDAD PERINATAL SEGÚN SEXO FETAL
}

\author{
Oscar Antonio Limay Ríos ${ }^{1}$, Maria del Carmen Comettant Puente ${ }^{2}$, Walter Castillo Urquiaga ${ }^{3}$, \\ Mario Zarate Girao ${ }^{3}$, Erasmo Huertas Tachino ${ }^{3}$, Jaime Ingar Pinedo ${ }^{3}$, Walter Ventura Laveriano ${ }^{3}$.
}

\begin{abstract}
RESUMEN
Objetivos. Determinar el riesgo de muerte en recién nacidos con diagnóstico de restricción de crecimiento intrauterino selectivo (RCIUs) en embarazo gemelar según el sexo. Materiales y métodos. Estudio analítico, transversal, retrospectivo. La muestra de recién nacidos con diagnóstico de RCIU fue seleccionada en el periodo comprendido entre el 21 de febrero del 2011 al 24 de noviembre del 2014 en el Instituto Nacional Materno Perinatal en donde se incluyeron a 60491 recién nacidos, de los cuales 58629 corresponden a embarazos únicos, 1790 de embarazos gemelares (683 recién nacidos con RCIUs; 335 de sexo femenino y 348 de sexo masculino) y 72 de embarazos triples. Resultados. La muerte perinatal para recién nacidos con RCIU según tipo de embarazo fue de 1,1\% en embarazos únicos, $2,1 \%$ en embarazo gemelar y $4,3 \%$ en embarazo triple. El Odds ratio (OR) de muerte perinatal para embarazo gemelar del mismo sexo respecto a embarazo gemelar de diferente sexo es de 4. El OR de muerte perinatal en gemelos con RCIUs sin diferenciar el sexo respecto a recién nacidos únicos es de 1,9. E OR de muerte perinatal en gemelos con RCIUs del mismo sexo respecto a recién nacidos únicos es de 4,4, en gemelos con RCIUs del mismo sexo masculino es de 5,5 y en gemelos con RCIUs del mismo sexo femenino es de 3,6. El OR de muerte perinatal en gemelos con RCIUs de diferente sexo respecto a recién nacidos únicos es de 0,5. El OR de muerte perinatal en recién nacidos de embarazo triple respecto a recién nacidos únicos es de 3,8. Conclusiones. La muerte perinatal es mayor en embarazos múltiples respecto a embarazos únicos. El riesgo de muerte perinatal es cuatro veces mayor en embarazo gemelar del mismo sexo que en embarazo gemelar de diferente sexo. El riesgo de muerte perinatal es mayor en recién nacidos con RCIU del mismo sexo masculino en embarazo gemelar respecto al resto de los casos expuestos.
\end{abstract}

Palabras clave: Restricción de crecimiento intrauterino selectivo; muerte perinatal; sexo fetal (Fuente: DeCs BIREME).

\section{SELECTIVE RESTRICTION OF INTRA UTERINE GROWTH AND RISK OF PERINATAL MORTALITY ACCORDING TO FETAL SEX}

\begin{abstract}
Objectives. To determinate the risk of death in new-borns with diagnosis of Selective intrauterine growth restriction in twin pregnancy according sex. Material and methods. Analytic, cross sectional and retrospective. The sample of new-borns with diagnosis of selective intrauterine growth restriction (SIUGR) was selected in the period between February $21^{\text {th }}, 2011$ and November $24^{\text {th }}$, 2014 on the National Maternal Perinatal Institute where were included 60491 new-borns, of which 58629 corresponded to single pregnancies, 1790 to twin pregnancies (683 new-borns with SIUGR; 335 of feminine sex and 348 of masculine sex) and 72 to triple pregnancies. Results. Perinatal death in Selective intrauterine growth restriction (SIUGR) new-borns according to the type

https://orcid.org/0000-0002-2888-1394 ${ }^{3} 1 \%$ in twin pregnancies and $4,3 \%$ in triple pregnancies. The Odds ratio (OR) of perinatal death in twin pregnancies with same sex related to twin pregnancies with different sex is 4 . The OR of perinatal death in SIUGR twins without making any differences of sex related to single pregnancies new-borns is 1,9 . The OR of perinatal death in SIUGR twins with same sex related to single pregnancies is 4,4, in SIUGR twins of same masculine sex is 5,5 and in SIUGR twins of same feminine sex is 3,6 . The OR of perinatal death in SIUGR twins with different sex related to single pregnancies new-borns is 0,5 . The OR of perinatal death of new-borns from triple pregnancies related to single pregnancies new-borns is 3,8 . Conclusions. Perinatal death is higher in multiple pregnancies related to single ones. The risk of perinatal death is four times higher in twin pregnancies from same sex than twin pregnancies from different sex. The risk of perinatal death is higher in SIUGR new-borns with same masculine sex in twin pregnancies related to the rest of exposed cases.
\end{abstract}

Key words: Selective intrauterine growth restriction; Perinatal death; Fetal sex ( Source: MeSH NLM).

\section{INTRODUCCIÓN}

Las gestaciones gemelares monocoriales son conocidas por presentar en mayor frecuencia complicaciones graves y por ende un aumento sustancial en la morbimortalidad perinatal para ambos gemelos ${ }^{1-6}$.

La evaluación mediante una ecografía temprana para identificar anomalías estructurales y la determinación precisa de corionicidad es crucial para el adecuado cuidado y seguimiento del embarazo múltiple, al mismo tiempo, se necesita de un etiquetado preciso de los gemelos para asegurar que el mismo feto cumpla con el patrón de crecimiento que le corresponde ${ }^{7-10}$.

La restricción del crecimiento intrauterino selectivo (RCIUS) es una complicación importante de los embarazos monocoriales con una frecuencia de 10 al $15 \%$, con potencial riesgo de muerte fetal intrauterina o disfunción

Médico Gíneco Obstetra, Magíster en Salud Pública, Asistente del Servicio de Medicina Fetal del Instituto Nacional Materno Perinatal. Lima-Perú.

Interna de Obstetricia de la Universidad Nacional Mayor de San Marcos- Sede Docente Instituto Nacional Materno Perinatal. Lima-Perú.

Médico Gíneco Obstetra, Asistente del Servicio de Medicina Fetal del Instituto Nacional Materno Perinatal. Lima-Perú.

Recibido: 14-01-15 Aprobado: 12-08-15 


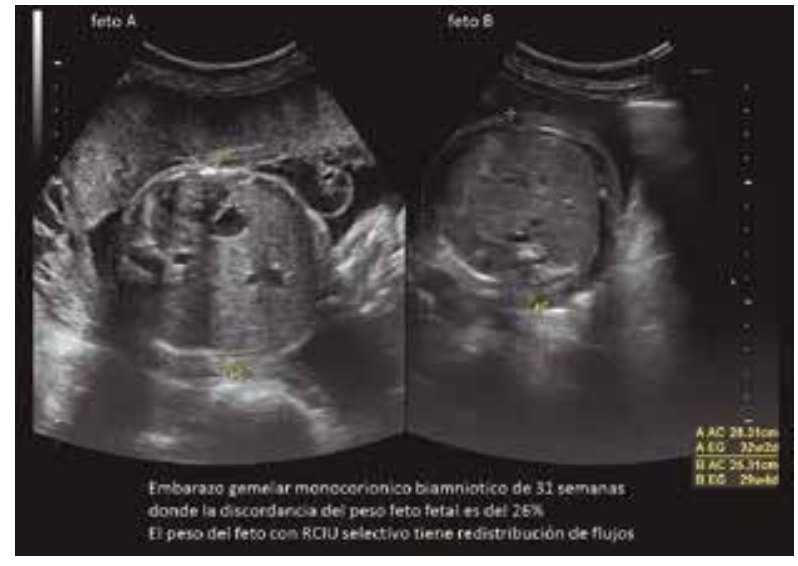

neurológica en ambos fetos ${ }^{1,6,8}$. El diagnostico ecográfico ha contribuido a la comprensión de la fisiopatología del RCIUS, a proponer una clasificación y a su vez, a interpretar una amplia variedad clínica para que en base a
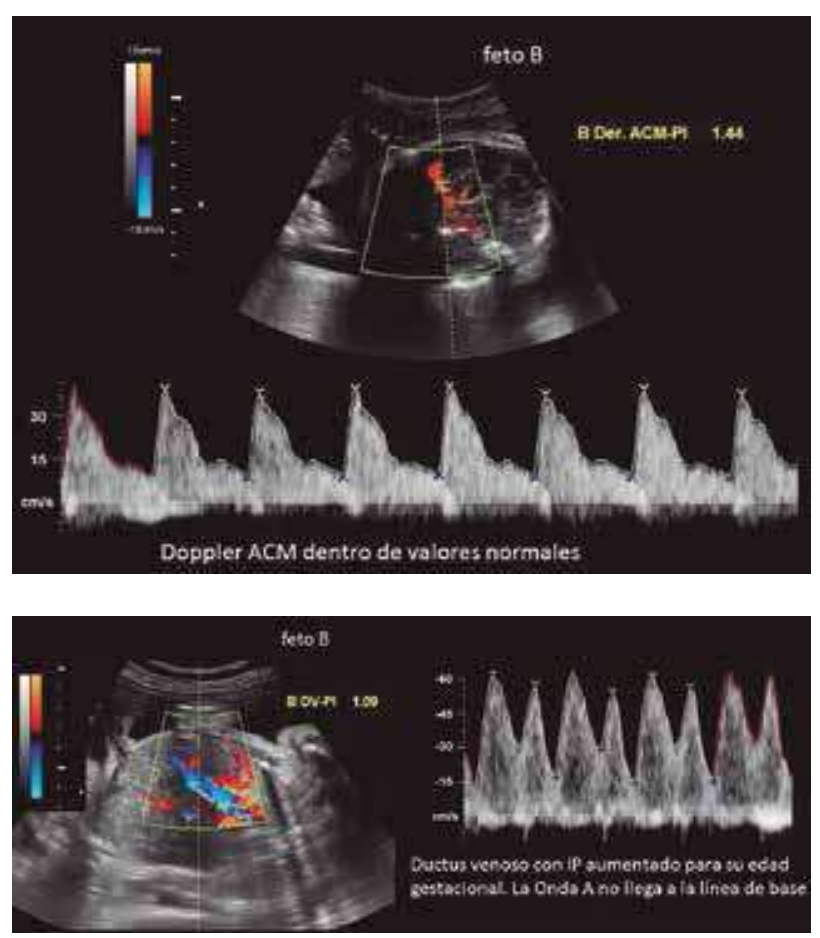

un pronóstico y estrategias optimas se realice un manejo clínico ${ }^{9-12}$.

La introducción de diagnóstico basado en estudios Doppler permite el correcto diagnóstico del trastorno y de su respectiva clasificación siendo el RCIU tipo I el que tiene un flujo diastólico normal y relativamente un buen resultado perinatal, el RCIU tipo II se define por presentar un flujo telediastólico persistente, ausente o retrogrado y está asociado con un alto riesgo de muerte intrauterina para el gemelo RCIU y/o parto prematuro; por último, el RCIU tipo III se define por la presencia intermitente de un flujo diastólico inverso o ausente y está asociado con un riesgo de muerte fetal en un $10-20 \%$ para el gemelo RCIU y de un $10-20 \%$ de riesgo de lesión neurológica para el gemelo no $\mathrm{RCIU}^{2,4,6-8,11-15}$.

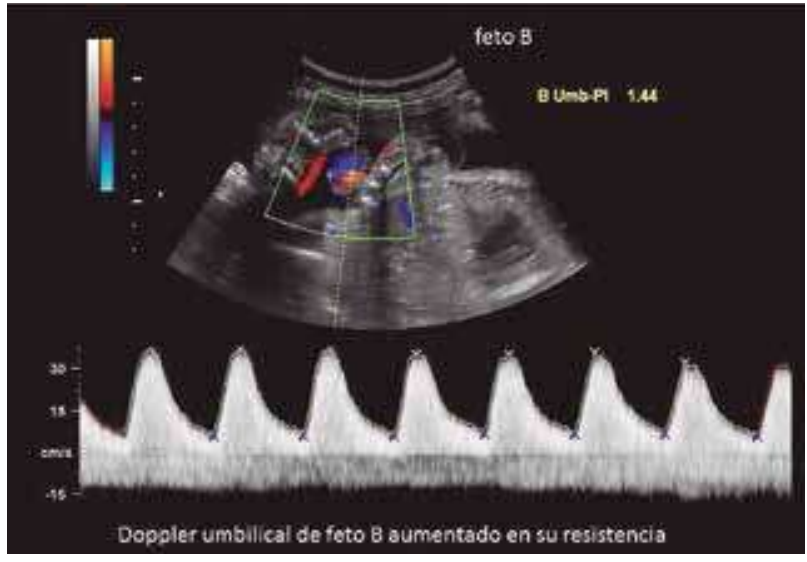

Múltiples estudios han concluido que los gemelos RCIUS contribuyen significativamente en las cifras de morbimortalidad perinatal y que éstas varían según su corionicidad, el momento de inicio de la patología y de la gravedad 11,13,15; sin embargo, no existen estudios que hagan una diferenciación en el pronóstico de estos RCIU según el sexo de los gemelos y que a su vez que esto implique un manejo clínico diferencial.

\section{MATERIALES Y MÉTODOS}

Se realizó un estudio analítico, retrospectivo entre el 21 de febrero del 2011 al 24 de noviembre del 2014 en el Instituto Nacional Materno Perinatal. Se incluyeron a los recién nacidos con diagnóstico de restricción de crecimiento intrauterino selectivo mediante ecografías realizadas en la Unidad de Medicina Fetal del Instituto cuyo nacimiento y seguimiento en el periodo perinatal fue realizado en la Institución. La población total a analizar fue de 60491 recién nacidos de los cuales 58529 corresponden a gestaciones únicas, 1790 a gestaciones dobles (683 recién nacidos con RCIUs de los cuales 335 corresponden al sexo femenino y 348 al sexo masculino) y 72 a gestaciones triples.

La información fue tomada de la base de datos de las ecografías desarrolladas en la Unidad de Medicina Fetal y de la base de datos de neonatos fallecidos durante los años de estudio; los datos fueron verificados mediante la revisión de múltiples registros de la Institución.

Se empleó la totalidad de la población que incluía con los criterios de inclusión para el análisis descriptivo, se excluyó a neonatos que no nacieron en la Institución o aquellos de los cuales se desconocía su resultado perinatal. Se procedió a la confección de una base de datos, conformada por una matriz estructurada en el programa Excel y se exporto la base de datos al programa SPSS v.22 para su análisis. Se realizó un análisis univariado para determinar frecuencias. Las variables a analizar fueron: tipo de gestaciones: único, gemelar o triple; resultado perinatal: recién nacido vivo o natimuerto; sexo: masculino o femenino. En el análisis bivariado 
se estableció como variables independientes el sexo asociado al resultado perinatal utilizando el riesgo relativo con sus intervalos de confianza al $95 \%$.

\section{RESULTADOS}

Durante el periodo de estudio hubieron un total de 60 491 nacimientos, de los cuales 58629 corresponden a embarazos únicos, 1790 de embarazos gemelares y 72 de embarazos triples (Tabla 1).

Tabla 1. Frecuencia de nacimientos según tipo de embarazo, 2011-2014

\begin{tabular}{cccc}
\hline $\begin{array}{c}\text { Tip Tipo de } \\
\text { gestación o de } \\
\text { gestación }\end{array}$ & $\begin{array}{c}\mathbf{N}^{\circ} \text { recién } \\
\text { nacidos }\end{array}$ & $\begin{array}{c}\mathbf{N}^{\circ} \\
\text { embarazos }\end{array}$ & Porcentaje (\%) \\
\hline Únicos & 58629 & 58629 & 98,45 \\
Gemelares & 1790 & 895 & 1,5 \\
\hline Triples & 72 & 28 & 0,05 \\
\hline Total & 60491 & 69552 & 100 \\
\hline
\end{tabular}

Se calculó la frecuencia y el porcentaje de los embarazos gemelares respecto a la totalidad de embarazos para cada año de estudio, encontrándose un total de $160(1,6 \%)$ para el año 2011, $286(1,8 \%)$ para el 2012, $224(1,3 \%)$ para el 2013 y 225 (1.4\%) para el año 2014; determinando un total de 895 embarazos y con un promedio de 1,5\% (Tabla 2).

Tabla 2. Frecuencia de embarazos por año de estudio

\begin{tabular}{ccc}
\hline Año & $\mathbf{N}^{\circ}$ Embarazos & $\%$ \\
\hline $\mathbf{2 0 1 1}$ & 160 & 1,6 \\
$\mathbf{2 0 1 2}$ & 286 & 1,8 \\
$\mathbf{2 0 1 3}$ & 224 & 1,3 \\
$\mathbf{2 0 1 4}$ & 225 & 1,4 \\
Total & 895 & 1,5 \\
\hline
\end{tabular}

Se determinó el resultado perinatal para cada tipo de embarazo encontrándose que en embarazos únicos hubieron $57983(98.9 \%)$ recién nacidos vivos y $646(1,1 \%)$ natimuertos; en embarazos gemelares hubieron 1753 $(97,9 \%)$ recién nacidos vivos y $37(2,1 \%)$ natimuertos; y en embarazos triples $69(95,8 \%)$ recién nacidos vivos y 3 $(4,3 \%)$ natimuertos (Tabla 3 y Gráfico 1$)$.

Tabla 3. Resultado perinatal según tipo de embarazo

\begin{tabular}{ccccc}
\hline \multirow{2}{*}{$\begin{array}{c}\text { Tipo de } \\
\text { embarazo }\end{array}$} & \multicolumn{4}{c}{ Resultado Perinatal } \\
\cline { 2 - 5 } & Recién nacido vivo & \multicolumn{2}{c}{ Natimuertos } \\
\cline { 2 - 5 } & $\mathrm{n}$ & $\%$ & $\mathrm{n}$ & $\%$ \\
\hline Únicos & 57983 & 98,9 & 646 & 1,1 \\
Gemelares & 1753 & 97,9 & 37 & 2,1 \\
Triples & 69 & 95,8 & 3 & 4,3 \\
Total & 59805 & & 686 & \\
\hline
\end{tabular}

Se estimó la tasa de muertes perinatales en embarazo gemelar según el sexo encontrándose que el $66,6 \%$ fueron del sexo masculino y el $33,3 \%$ fueron del sexo

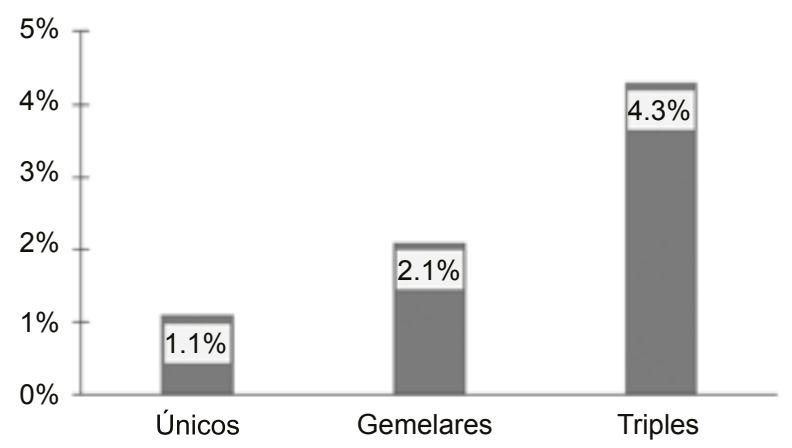

Grafico 1. Natimuertos según tipo de embarazo

femenino. Así mismo, se demostró que cuando hay un embarazo gemelar del mismo sexo hay un Odds ratio (OR) de 4 de mortalidad perinatal respecto a embarazo gemelar de diferente sexo (Tabla 4).

Tabla 4. Riesgo de muerte en recién nacidos de embarazo gemelar sin RCIUs

\begin{tabular}{cccc}
\hline Tipo de gemelar & Natimuertos & Vivos & Total \\
\hline Mismo sexo & 15 & 692 & 707 \\
Diferente sexo & 1 & 187 & 188 \\
Total & 16 & 879 & 895 \\
OR & & 4,0 & \\
\hline
\end{tabular}

Mediante el análisis bivariado se calculó el Odds ratio para gemelos natimuertos con RClUs sin diferenciar el sexo comparado con recién nacidos únicos siendo éste el valor de 1,9 lo mismo se hizo para gemelos natimuertos con RCIUs del mismo sexo comparados con recién nacidos únicos y la cifra del OR aumentó a 4,4; y para gemelos natimuertos con RCIUs de sexo diferente comparado con recién nacidos únicos encontrándose un OR de 0,5. Se ajustó el OR para gemelos natimuertos con RCIUs del mismo sexo masculino comparado con recién nacidos únicos y la cifra aumentó significativamente respecto a las anteriores siendo 5,5 el OR; sin embargo, para gemelos natimuertos con RCIUs del mismo sexo femenino comparado con recién nacidos únicos la cifra fue solo de 3,6. Por último, se calculó el OR para natimuertos en embarazos triples comparados con recién nacidos únicos siendo el valor 3,8 (Tabla 5-6 y Gráfico 2).

Tabla 5. Riesgo de muerte en recién nacidos con RCIUs en embarazo gemelar respecto a recién nacidos únicos sanos

\begin{tabular}{cccc}
\hline \multirow{2}{*}{$\begin{array}{c}\text { Recién nacidos gemelar } \\
\text { con RCIUs }\end{array}$} & \multicolumn{2}{c}{$\begin{array}{c}\text { Recién Nacidos únicos } \\
\text { sanos }\end{array}$} & \multirow{2}{*}{ OR } \\
\cline { 2 - 3 } & Vivos & Natimuertos & \\
\cline { 2 - 3 } & 57983 & 646 & \\
\hline Sin diferencia de sexo & 1753 & 37 & 1,9 \\
\hline Igual sexo & 690 & 35 & 4,4 \\
\hline Igual sexo femenino & 338 & 14 & 3,6 \\
\hline Igual sexo masculino & 354 & 23 & 5,5 \\
\hline Diferente sexo & 187 & 1 & 0,5 \\
\hline
\end{tabular}


Tabla 6. Riesgo de muerte en recién nacidos de embarazo triple respecto a recién nacidos únicos

\begin{tabular}{cccc}
\hline & Natimuertos & Vivos & Total \\
\hline Triples & 3 & 69 & 72 \\
Rn únicos & 646 & 57983 & 58629 \\
Total & 649 & 58052 & 58701 \\
OR & & 3,8 & \\
\hline
\end{tabular}

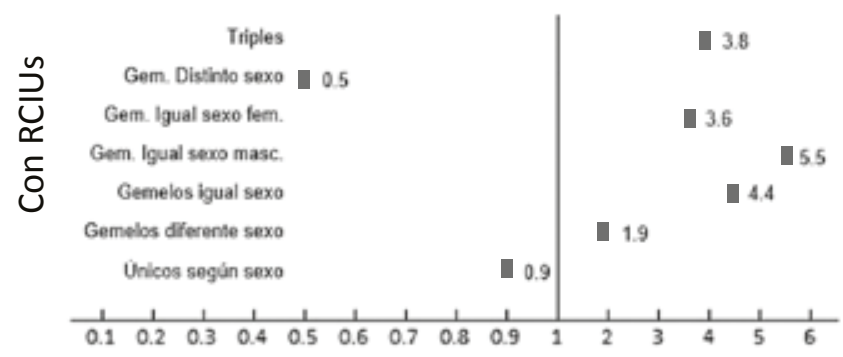

Gráfico 2. Riesgo de muerte según embarazo en recién nacidos con RCIUs

\section{DISCUSIÓN}

La morbilidad y mortalidad perinatal en recién nacidos con diagnóstico de restricción de crecimiento intrauterino es un asunto de gran importancia que compromete tanto al campo de la ginecología como al de neonatología por lo que el diagnóstico oportuno, pronostico y tratamiento de éstos deberá involucrar la participación en equipo de ambos profesionales ${ }^{16}$.

Múltiples estudios como Valski $\mathrm{D}^{1}$, Ishii $\mathrm{K}^{15}$, Weisz $\mathrm{B}^{16}$, han demostrado que los recién nacidos con diagnóstico de RCIU selectivo tienen un riesgo mayor de morbimortalidad perinatal de $4 \%$ respecto a aquellos que no presentan esta patología.

Es conocido además que en embarazo gemelar la corionicidad juega un papel importante en el diagnóstico de RCIU selectivo y en su resultado perinatal. Gao $Y^{5}$ demostró en el 2011 que la monocorionicidad es un factor de riesgo para presentar RCIU selectivo, mayor daño neurológico neonatal y mayor muerte perinatal. Moises $\mathrm{K}^{3}$ reportó además que los gemelos monocoriales presentan con mayor frecuencia el síndrome de transfusión feto fetal, restricción de crecimiento intrauterino selectivo y perfusión arterial gemelar invertida. Yu $\mathrm{H}^{6}$ en el 2013 demostró que los recién nacidos monocoriales con RCIU selectivo tenían una incidencia de muerte perinatal de un $28 \%$, parto prematuro de $69 \%$, bajo peso al nacer de $33 \%$ y asfixia al nacer de $25 \%$.

El diagnostico ecográfico de restricción de crecimiento intrauterino selectivo ha contribuido a la comprensión de su fisiopatología y a determinar una clasificación para proponer un manejo clínico especifico. Kowalska Jasiecka $\mathrm{J}^{14}$ menciona en su estudio que la introducción del diagnóstico basado en estudios Doppler permite el correcto diagnóstico, monitoreo fetal, pronóstico y como consecuencia determinar estrategias óptimas y proponer la mejor intervención terapéutica. D' Antonio $F^{11}$ y Sueters $\mathrm{M}^{13}$ proponen que un monitoreo frecuente sumado al uso de herramientas de diagnóstico adecuadas y un conocimiento especializado contribuye a una reducción en el riesgo de morbimortalidad perinatal en los casos de restricción de crecimiento intrauterino.

Sin embargo, a pesar de todos estos estudios mencionados anteriormente, actualmente no existe evidencia de que el sexo sea considerado un factor de riesgo de morbimortalidad perinatal en los gemelos con restricción de crecimiento intrauterino por lo que en el presente estudio tiene como objetivo demostrar cual es el riesgo de muerte perinatal en recién nacidos con restricción de crecimiento intrauterino selectivo de embarazo gemelar según su sexo.

Los resultados demostraron que en los embarazos gemelares ser del mismo sexo implica tener 4 veces más riesgo de mortalidad perinatal en relación a aquellos que son de diferente sexo. Al mismo tiempo se realizó el mismo análisis pero comparando a los recién nacidos con restricción de crecimiento intrauterino en embarazo gemelar sin diferenciar el sexo con recién nacidos únicos encontrándose un riesgo de muerte perinatal de 1,9 sin embargo cuando estos eran del mismo sexo el riesgo de muerte aumentaba a 4,4 y muy por el contrario cuando eran de diferente sexo el riesgo era solo de 0,5 pudiendo ser considerado como un factor protector de muerte perinatal comparado con recién nacidos únicos. Lo que más llamo la atención de los resultados fue cuando se descubrió que los recién nacidos de embarazo gemelar con el mismo sexo masculino tienen 5,5 veces más riesgo de muerte en relación a los recién nacidos únicos, mientras que cuando son del mismo sexo femenino el riesgo fue de 3,6 , llegando a ser incluso mayor cuando la comparación se hacía entre recién nacidos de embarazos triples y recién nacidos únicos cuyo riesgo era solo de 3,8 .

Como conclusión la importancia de este estudio radica en que los resultados demostraron que en los casos diagnosticados con restricción de crecimiento selectivo en embarazos gemelares se debe de tener en cuenta que el sexo es un factor importante como predictor del resultado perinatal, específicamente de muerte perinatal, necesitando por ello un manejo especifico los fetos del mismo sexo, especialmente si ambos son del sexo masculino, puesto que estos tienen un riesgo de muerte mayor en comparación al resto de los casos.

Finalmente, se propone investigar cuales serían las posibles causas de por qué el sexo es considerado como un factor de riesgo de muerte perinatal en recién nacidos con restricción de crecimiento intrauterino y por qué ser del sexo masculino tiene peor pronóstico perinatal que ser del sexo femenino. 


\section{REFERENCIAS BIBLIOGRÁFICAS}

1. Valsky DV, Eixarch E, Martinez JM. Selective intrauterine growth restriction in monochorionic twins: pathophysiology, diagnostic approach and management dilemmas. Semin Fetal Neonatal Med. 2010 Dec; 15(6):342-8.

2. Sueters $M$, Oepkes $D$. Diagnosis of twin-to-twin transfusion syndrome, selective fetal growth restriction, twin anaemiapolycythaemia sequence, and twin reversed arterial perfusion sequence. Best Pract Res Clin Obstet Gynaecol. 2014 Feb; 28(2):215-26.

3. Moises KY, Kugler L, Jones T. Contemporary management of complicated monochorionic twins. J Obstet Gynecol Neonatal Nurs. 2012 May-Jun; 41(3):434-44; quiz 445-6.

4. Torres-Torres C, Pérez-Borbón G, Benavides-Serralde JA. Prevalence and complications of monochorionic diamniotic twin pregnancy. Ginecol Obstet Mex. 2010 Mar; 78 (3):181-6.

5. Gao Y, He Z, Luo Y. Selective and non-selective intrauterine growth restriction in twin pregnancies: high-risk factors and perinatal outcome. Arch Gynecol Obstet. 2012 Apr; 285 (4):973-8.

6. Yu HY, Lau T, Sahota DS. Perinatal outcome of monochorionic twin pregnancies. Zhonghua Fu Chan Ke Za Zhi. 2013 Jun; 48 (6):405-10. Chinese.

7. Ishii K, Murakoshi $T$, Sago $H$. Adverse outcome in monochorionic twins with selective intrauterine fetal growth restriction in the presence of abnormal umbilical artery Doppler and severe oligohydramnios. J Obstet Gynaecol Res. 2012 Oct; 38 (10):1271.
8. Inklaar MJ, van Klink JM, Stolk TT. Cerebral injury in monochorionic twins with selective intrauterine growth restriction: a systematic review. Prenat Diagn. 2014 Mar; 34(3):205-13.

9. Simpson LL. Ultrasound in twins: dichorionic and monochorionic. Semin Perinatol. 2013 Oct; 37(5):348-58.

10. Moise KJ Jr. Surveillance of monochorionic twins. Am J Perinatol. 2014 Sep;31

11. D'Antonio F, Bhide A. Early pregnancy assessment in multiple pregnancies. Best Pract Res Clin Obstet Gynaecol. 2014 Feb; 28(2):201-14.

12. Tan TY, Yeo GS. Intrauterine growth restriction. Curr Opin Obstet Gynecol. 2005 Apr; 17(2):135-42.

13. Kowalska-Jasiecka J, Ropacka-Lesiak M, Breborowicz G. Selective intrauterine growth restriction in monochorionic twin pregnancies. Ginekol Pol. 2012 Aug; 83(8):618-21.

14. Gratacós E, Lewi L, Muñoz B, Acosta-Rojas R. A classification system for selective intrauterine growth restriction in monochorionic pregnancies according to umbilical artery Doppler flow in the smaller twin. Ultrasound Obstet Gynecol. 2007 Jul; 30(1):28-34.

15. Ishii K, Murakoshi T, Hayashi S. Ultrasound predictors of mortality in monochorionic twins with selective intrauterine growth restriction. Ultrasound Obstet Gynecol. 2011 Jan; 37(1):22-6.

16. Weisz B, Hogen L, Yinon Y. Perinatal outcome of monochorionic twins with selective IUGR compared with uncomplicated monochorionic twins. Twin Res Hum Genet. 2011 Oct; 14(5):457-62.

\section{ORCID iDs}

Oscar Antonio Limay Ríos Walter Castillo Urquiaga Erasmo Huertas Tacchino Jaime Ingar Pinedo Walter Ventura Laveriano https://orcid. org/0000-0001-6012-3705

https://orcid.org/0000-0002-1054-7398

https://orcid.org/0000-0002-9851-8419

https://orcid.org/0000-0001-6472-7899

https://orcid.org/0000-0002-2888-1394 